\title{
Universiteit
}

Leiden

The Netherlands

\section{Permanent tuning of quantum dot transitions to degenerate} microcavity resonances

Gudat, J.; Bonato, C.; Nieuwenburg, E. van; Thon, S.M.; Kim, H.; Petroff, P.M.; ... ; Bouwmeester, D.

\section{Citation}

Gudat, J., Bonato, C., Nieuwenburg, E. van, Thon, S. M., Kim, H., Petroff, P. M., ... Bouwmeester, D. (2011). Permanent tuning of quantum dot transitions to degenerate microcavity resonances. Applied Physics Letters, 98(12), 121111. doi:10.1063/1.3569587

Version: $\quad$ Not Applicable (or Unknown)

License: $\quad$ Leiden University Non-exclusive license

Downloaded from: https://hdl.handle.net/1887/65891

Note: To cite this publication please use the final published version (if applicable). 


\section{Permanent tuning of quantum dot transitions to degenerate microcavity resonances}

Jan Gudat, Cristian Bonato, Evert van Nieuwenburg, Susanna Thon, Hyochul Kim, Pierre M. Petroff, Martin P. van Exter, and Dirk Bouwmeester

Citation: Appl. Phys. Lett. 98, 121111 (2011); doi: 10.1063/1.3569587

View online: https://doi.org/10.1063/1.3569587

View Table of Contents: http://aip.scitation.org/toc/apl/98/12

Published by the American Institute of Physics

\section{Articles you may be interested in}

Tuning micropillar cavity birefringence by laser induced surface defects

Applied Physics Letters 95, 251104 (2009); 10.1063/1.3276550

Independent tuning of quantum dots in a photonic crystal cavity

Applied Physics Letters 95, 243107 (2009); 10.1063/1.3275002

Independent electrical tuning of separated quantum dots in coupled photonic crystal cavities

Applied Physics Letters 99, 161102 (2011); 10.1063/1.3651491

Deterministic nanoassembly of a coupled quantum emitter-photonic crystal cavity system Applied Physics Letters 98, 193103 (2011); 10.1063/1.3571437

Fiber-connectorized micropillar cavities

Applied Physics Letters 97, 131113 (2010); 10.1063/1.3493187

Strong coupling through optical positioning of a quantum dot in a photonic crystal cavity

Applied Physics Letters 94, 111115 (2009); 10.1063/1.3103885

\section{Conference Proceedings}

Get $30 \%$ off all print proceedings!
Enter Promotion Code PDF-30 at checkout

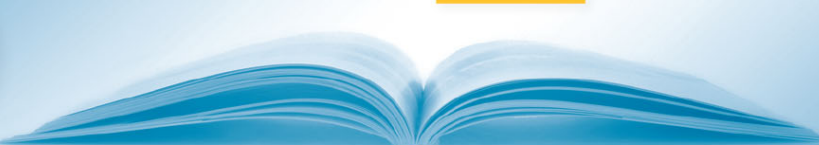




\title{
Permanent tuning of quantum dot transitions to degenerate microcavity resonances
}

\author{
Jan Gudat, ${ }^{1}$ Cristian Bonato, ${ }^{1, a)}$ Evert van Nieuwenburg, ${ }^{1}$ Susanna Thon, ${ }^{2}$ Hyochul Kim, ${ }^{2}$ \\ Pierre M. Petroff, ${ }^{2}$ Martin P. van Exter, ${ }^{1}$ and Dirk Bouwmeester ${ }^{1,2}$ \\ ${ }^{1}$ Huygens Laboratory, Leiden University, P.O. Box 9504, 2300 RA Leiden, The Netherlands \\ ${ }^{2}$ University of California Santa Barbara, Santa Barbara, California 93106, USA
}

(Received 11 February 2011; accepted 1 March 2011; published online 23 March 2011)

\begin{abstract}
We demonstrate a technique for achieving spectral resonance between a polarization-degenerate micropillar cavity mode and an embedded quantum dot transition. Our approach is based on a combination of isotropic and anisotropic tensile strain effected by laser-induced surface defects, thereby providing permanent tuning. Such a technique is a prerequisite for the implementation of scalable quantum information schemes based on solid-state cavity quantum electrodynamics.

(C) 2011 American Institute of Physics. [doi:10.1063/1.3569587]
\end{abstract}

Single self-assembled quantum dots (QDs) embedded in microcavities are interesting systems for quantum information applications. Cavity-induced Purcell enhancement of the emitter spontaneous emission rate has been exploited to demonstrate efficient and reliable single photon sources. ${ }^{1-3}$ Moreover, quantum information schemes employing cavity quantum electrodynamics with QDs coupled to semiconductor microcavities have been proposed and implemented. ${ }^{4-8}$ Such system would provide a scalable platform for hybrid quantum information protocols, in which photonic qubits are used for long-distance transmission and matter qubits for local storage and processing. ${ }^{9,10}$

Several quantum information applications require a polarization-degenerate cavity mode that is spectrally resonant with a specific QD optical transition. ${ }^{7,8}$ Polarizationdegeneracy is needed in order to transfer an arbitrary polarization state of a photon to the spin of a single electron confined in the dot, or vice versa. In the case of micropillar cavities, due to residual strain in the structure or small shape asymmetries, the fundamental cavity mode often consists of two linearly-polarized submodes, energy split by an amount $\Delta E$. An important issue to note is the fact that the optical properties of a self-assembled QD strongly depend on its specific size and local strain, neither of which is deterministically controllable in the growth process. Therefore postfabrication tuning techniques are crucial to achieving exact spectral resonance.

The most flexible tuning technique is Stark-shifting: embedding the dots in a diode structure and applying a voltage leads to a shift in the optical transition frequency by the quantum confined Stark effect. ${ }^{11}$ Such shifts can be finely tuned to a limited range of a few hundred microelectron volt, making the technique most effective in combination with some other coarse tuning procedures, such as temperature or strain. Temperature tuning, of either the whole sample ${ }^{12}$ or a local spot ${ }^{13}$ is an effective approach, with energy shifts on the order of 1-2 meV reported in the literature. The temperature can, however, only be adjusted in the range of about 4-50 K: at higher temperatures the dot luminescence quenches. Moreover, if one is interested in the spin of a single electron in the $\mathrm{QD}$, it is crucial to keep the tempera-

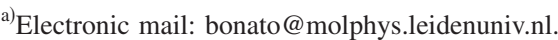

ture below $30 \mathrm{~K}$, in order to avoid reducing the spin relaxation time. ${ }^{14}$

Strain-tuning, via piezoelectric actuators or mechanical tips, has also been extensively investigated. ${ }^{15-17}$ Recently, it was shown that strain control by means of laser-induced surface defects can be used to fine-tune the optical properties of semiconductor microcavities. ${ }^{18,19}$ By focusing a strong laser beam on a small spot, far away from the cavity center to preserve the optical quality of the device, the local birefringence can be modified. Here we show that, by a controlled combination of anisotropic and isotropic strain, one can simultaneously get a polarization-degenerate cavity and tune a dot optical transition into resonance with the cavity mode. Since the defects are permanent, no external tuning equipment is needed during an experiment, and this makes our technique ideal for scalability purposes.

We investigated a sample with QDs embedded in micropillar cavities, grown by molecular-beam epitaxy on a GaAs [100] substrate. The microcavity consists of two distributed Bragg reflector (DBR) mirrors, made by alternating $\lambda / 4$ layers of $\mathrm{GaAs}$ and $\mathrm{Al}_{0.9} \mathrm{Ga}_{0.1}$ As. Between the mirrors, the active $\lambda$-GaAs layer contains embedded InGaAs/GaAs selfassembled QDs and sits underneath an AlAs oxidation layer. Trenches are etched down to the bottom DBR and the sample is placed in a steam oven to define an $\mathrm{AlO}_{x}$ oxidation front in the AlAs layer, providing transverse optical-mode confinement which results in high quality factors. ${ }^{20}$ Using micropillars defined by trench shapes, intracavity electrical gating of multiple devices is possible by the fabrication of a PIN-diode structure (see Fig. 1 for a sample diagram).

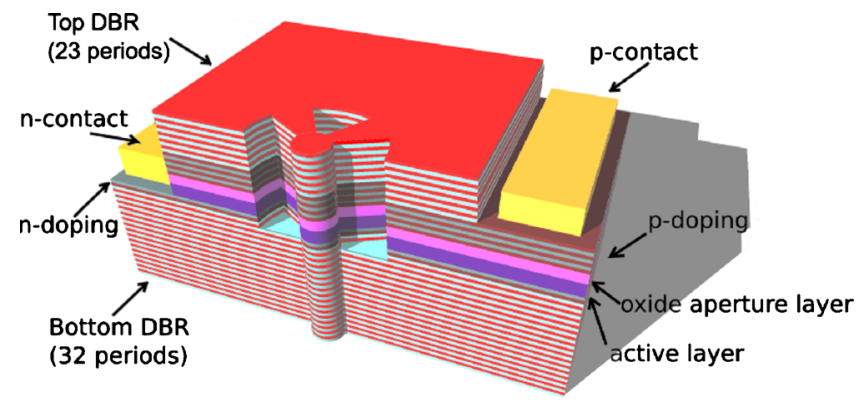

FIG. 1. (Color online) Sketch of the micropillar structure used in the experiments. 


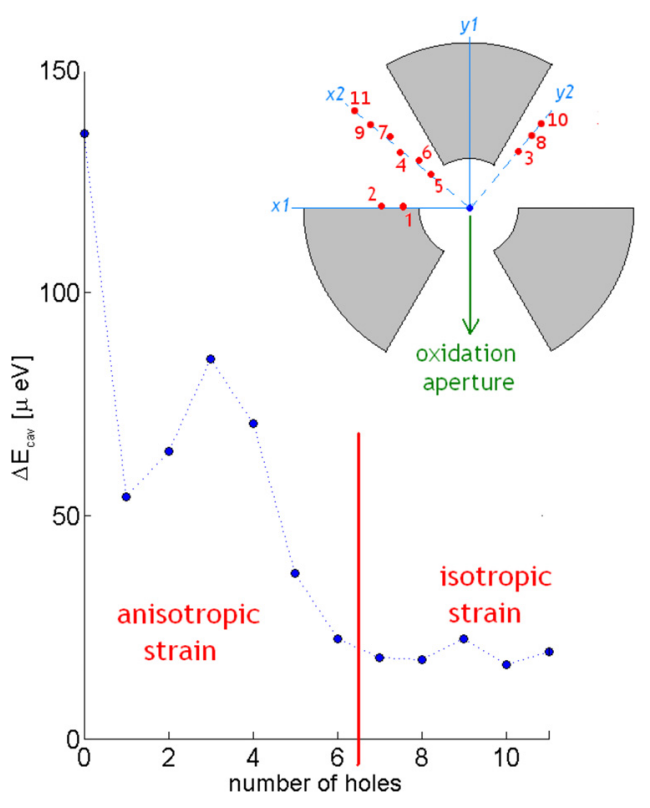

FIG. 2. (Color online) Frequency splitting of the two orthogonally-polarized submodes of the fundamental cavity mode as a function of the burnt holes.

Defects are created on the sample surface by a laser beam (about $100 \mathrm{~mW} / \mu \mathrm{m}^{2}, \lambda=532 \mathrm{~nm}$ ) tightly focused on the structure for about $30 \mathrm{~s}$ by a high-numerical aperture $(\mathrm{NA}=0.6)$ aspheric lens $L_{1}$, with focal length $f_{0}=4.2 \mathrm{~mm}$. The material is locally melted and evaporated, leaving a hole which is approximately $2 \mu \mathrm{m}$ wide and at least $2 \mu \mathrm{m}$ deep. The whole process is performed in a helium-flow cryostat, at a temperature of $4 \mathrm{~K}$.

The first step consists of reducing the fundamental cavity mode to polarization-degeneracy, following the procedure described in Bonato et al. ${ }^{19}$ The built-in strain can be compensated by applying anisotropic strain, through holes burnt at proper positions. The direction of the original built-in strain is however unknown, so one must use a trial-and-error procedure, illustrated in Fig. 2. We first start burning a hole at a random orientation, for example along the direction labeled in the figure as $x_{1}$. If the splitting gets larger, we move to the orthogonal direction. If the splitting decreases, we keep burning holes until the splitting stops decreasing. In the example shown in Fig. 2, the first hole reduces $\Delta E$ from $140 \pm 4 \mu \mathrm{eV}$ to $54 \pm 1 \mu \mathrm{eV}$, but a second one slightly increases it. This is an indication that all the strain along that particular direction was compensated. We repeat the same procedure on a reference system rotated by $45^{\circ}$ with respect to $\left[x_{1}, y_{1}\right]$. In the example, we start burning the third hole along $y_{2}$, which increases the splitting to $\Delta E$ $=82.6 \pm 0.4 \mu \mathrm{eV}$. Therefore we switch to the orthogonal direction $x_{2}$. Burning holes along this direction reduces $\Delta E$ to around $15 \mu \mathrm{eV}$. The procedure can be further iterated along directions in between $x_{1}$ and $x_{2}$ and generally leads to splittings smaller than the mode linewidth (in our system about $50 \mu \mathrm{eV}$ ), which is the requirement for quantum information experiments. No appreciable change in the cavity quality factor was observed.

Strain affects the optical transitions of the QDs as well. In Fig. 3, we show plots of voltage-resolved photoluminescence from the same microcavity analyzed in Fig. 2. We pump the sample nonresonantly with about $1 \mu \mathrm{W} / \mu \mathrm{m}^{2}$ laser beam at $785 \mathrm{~nm}$, above the GaAs bandgap, and we spec-

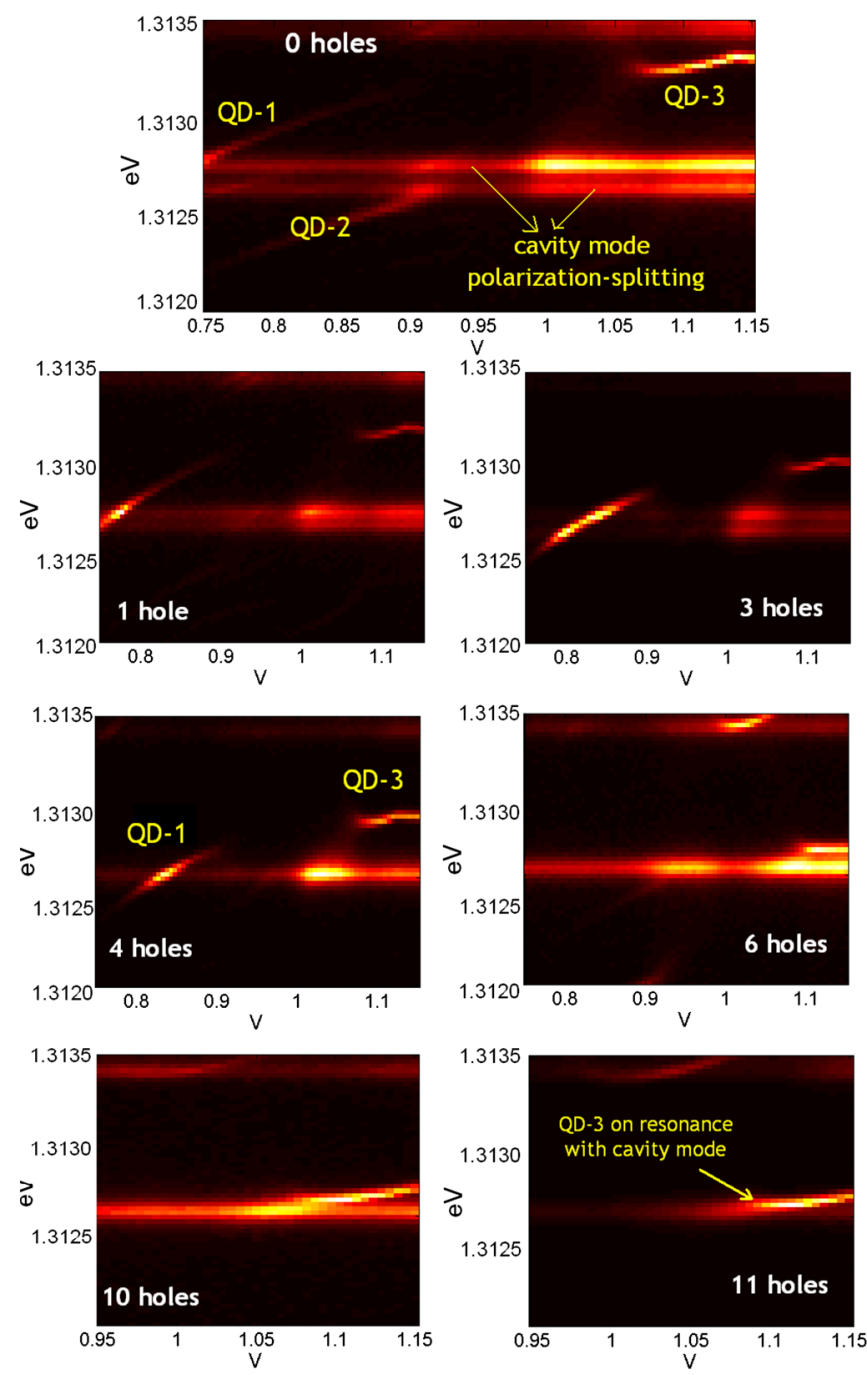

FIG. 3. (Color online) Voltage-resolved photoluminescence plots for the holes described in Fig. 2. Originally, the cavity mode is nondegenerate (splitting around $140 \mu \mathrm{eV}$ ) and QD-3 is around $0.5 \mathrm{meV}$ detuned to the blue-side of the cavity mode. Burning 6 holes reduces the splitting to about $15 \mu \mathrm{eV}$ and QD-3 is about $0.1 \mathrm{meV}$ detuned. Applying isotropic strain, by burning pair of holes along orthogonal direction, the dot can be brought into resonance with the cavity mode, without destroying the mode degeneracy (see plot for 11 holes, bottom right). See Fig. 2 for the position of the holes.

trally resolve the photoluminescence with a spectrometer (resolution $25 \mu \mathrm{eV} /$ pixel). Scanning the voltage of the microstructure PIN-diode, different charged states of the dot can be selected ${ }^{21}$ and the frequency of the optical transitions can be tuned by the Stark effect. ${ }^{11}$ The flat lines in the plots correspond to the fixed frequency emission of the fundamental cavity mode, split into two orthogonally-polarized submodes. The Stark-shifting lines correspond to QD optical transitions.

The effect of laser-induced defects is always a redshift in the optical transition, independent of the actual position of the hole. The shift in the dot transition is generally much larger than the corresponding shift in the cavity mode, and from a sample of more than one hundred holes burnt, the ratio of the shifts was found to be on average 5:1. These findings can be explained with a simple model. ${ }^{22}$ The fact that the optical transition always redshifts suggests that by burning holes we effectively apply tensile strain to the structure. This could be explained by assuming that, by removing material, we release some compressive strain that pre-exists 
due to lattice-mismatch in the dot. Such tensile strain affects the band-structure both of the InAs dot material and of the bulk surrounding GaAs, reducing the InAs energy gap and the width of the confining potential well. The change in the band-structure profiles can be shown to be independent of the direction of the strain in the plane of the dot. ${ }^{22}$

The difference in the way the cavity mode and the dot transition are affected by hole-burning can be exploited to tune a QD transition into resonance with a polarizationdegenerate cavity. In Fig. 3 one can see that, while burning the first six holes, needed to reduce the splitting $\Delta E$, the optical transitions of the dots redshift, so that the transitions labeled as QD-1 and QD-2, originally resonant with the nondegenerate fundamental cavity mode, tune out of resonance. After burning six holes we have a polarization-degenerate cavity mode, with a QD transition (labeled QD-3) about $100 \mu \mathrm{eV}$ detuned on the blue-side. Now the challenge is to shift this transition into resonance, without perturbing the cavity mode degeneracy. This can be done by applying isotropic strain: we can burn sets of two holes at orthogonal directions, for example, one along $x_{2}$ and the other along $y_{2}$, at the same distance from the center. This leaves the splitting $\Delta E$ unaltered while redshifting the dot transition. The results are shown in the bottom two pictures in Fig. 3, corresponding to the tenth and eleventh hole burnt. The dot is finally on resonance and the fundamental cavity mode splitting is $13 \pm 1 \mu \mathrm{eV}$ (right side of Fig. 2, for holes 7-11).

In conclusion, we demonstrated a tuning technique for micropillar cavities with embedded QDs, which allows us to obtain polarization-degenerate micropillars with a QD transition on resonance. Our technique is a crucial prerequisite for the implementation of scalable quantum information systems involving photon polarization and the spin of a single carrier trapped in the dot.

This work was supported by the NSF Grant No. 0901886, the Marie-Curie Award No. EXT-CT-2006-042580, and FOM\NWO Grant No. 09PR2721-2. We thank Andor for the charge-coupled device camera.

${ }^{1}$ B. Lounis and M. Orrit, Rep. Prog. Phys. 68, 1129 (2005).

${ }^{2}$ S. Strauf, N. G. Stoltz, M. T. Rakher, L. A. Coldren, P. M. Petroff, and D.
Bouwmeester, Nat. Photonics 1, 704 (2007).

${ }^{3}$ S. Reitzenstein and A. Forchel, J. Phys. D: Appl. Phys. 43, 033001 (2010)

${ }^{4}$ A. Imamoglu, D. D. Awschalom, G. Burkard, D. P. DiVincenzo, D. Loss, M. Sherwin, and A. Small, Phys. Rev. Lett. 83, 4204 (1999).

${ }^{5}$ J. P. Reithmaier, G. Sek, A. Loffler, C. Hofmann, S. Kuhn, S. Reitzenstein, L. V. Keldysh, V. D. Kulakovskii, T. L. Reinecke, and A. Forchel, Nature (London) 432, 197 (2004).

${ }^{6}$ M. T. Rakher, N. G. Stoltz, L. A. Coldren, P. M. Petroff, and D. Bouwmeester, Phys. Rev. Lett. 102, 097403 (2009).

${ }^{7}$ C. Y. Hu, W. J. Munro, J. L. O’Brien, and J. G. Rarity, Phys. Rev. B 80, 205326 (2009).

${ }^{8}$ C. Bonato, F. Haupt, S. S. R. Oemrawsingh, J. Gudat, D. Ding, M. P. van Exter, and D. Bouwmeester, Phys. Rev. Lett. 104, 160503 (2010).

${ }^{9}$ J. I. Cirac, P. Zoller, H. J. Kimble, and H. Mabuchi, Phys. Rev. Lett. 78, 3221 (1997).

${ }^{10}$ S. J. van Enk, J. I. Cirac, and P. Zoller, Phys. Rev. Lett. 78, 4293 (1997).

${ }^{11}$ P. W. Fry, I. E. Itskevich, D. J. Mowbray, M. S. Skolnick, J. J. Finley, J. A. Barker, E. P. O'Reilly, L. R. Wilson, I. A. Larkin, P. A. Maksym, M. Hopkinson, M. Al-Khafaji, J. P. R. David, A. G. Cullis, G. Hill, and J. C. Clark, Phys. Rev. Lett. 84, 733 (2000).

${ }^{12}$ A. Kiraz, P. Michler, C. Becher, B. Gayral, A. Imamoglu, L. Zhang, E. Hu, W. V. Schoenfeld, and P. M. Petroff, Appl. Phys. Lett. 78, 3932 (2001).

${ }^{13}$ A. Faraon, D. Englund, I. Fushman, J. Vučković, N. Stoltz, and P. Petroff, Appl. Phys. Lett. 90, 213110 (2007).

${ }^{14}$ M. Paillard, X. Marie, P. Renucci, T. Amand, A. Jbeli, and J. M. Gérard, Phys. Rev. Lett. 86, 1634 (2001).

${ }^{15}$ C. Obermüller, A. Deisenrieder, G. Abstreiter, K. Karrai, S. Grosse, S. Manus, J. Feldmann, H. Lipsanen, M. Sopanen, and J. Ahopelto, Appl. Phys. Lett. 75, 358 (1999).

${ }^{16}$ T. Zander, A. Herklotz, S. Kiravittaya, M. Benyoucef, F. Ding, P. Atkinson, S. Kumar, J. D. Plumhof, K. Dörr, A. Rastelli, and O. G. Schmidt, Opt. Express 17, 22452 (2009).

${ }^{17}$ G. W. Bryant, M. Zieliński, N. Malkova, J. Sims, W. Jaskólski, and J. Aizpurua, Phys. Rev. Lett. 105, 067404 (2010).

${ }^{18}$ A. K. Jansen van Doorn, M. P. van Exter, and J. P. Woerdman, Appl. Phys. Lett. 69, 1041 (1996).

${ }^{19}$ C. Bonato, D. Ding, J. Gudat, S. Thon, H. Kim, P. M. Petroff, M. P. van Exter, and D. Bouwmeester, Appl. Phys. Lett. 95, 251104 (2009).

${ }^{20}$ N. G. Stoltz, M. Rakher, S. Strauf, A. Badolato, D. D. Lofgreen, P. M. Petroff, L. A. Coldren, and D. Bouwmeester, Appl. Phys. Lett. 87, 031105 (2005).

${ }^{21}$ R. J. Warburton, C. Schaeflein, D. Haft, F. Bickel, A. Lorke, K. Karrai, J. M. Garcia, M. Schoenfeld, and P. M. Petroff, Nature (London) 405, 926 (2000)

${ }^{22}$ C. Bonato, E. van Nieuwenburg, J. Gudat, S. Thon, H. Kim, M. van Exter, and D. Bouwmeester, "Strain-tuning of quantum dot optical transitions via laser-induced surface defects" (unpublished). 